\title{
Critical dynamics of diluted relaxational models coupled to a conserved density (diluted model $\mathrm{C}$ )
}

\author{
M. Dudka \\ Institute for Condensed Matter Physics, National Academy of Sciences of Ukraine, UA-79011 Lviv, Ukraine and \\ Institute for Theoretical Physics, University of Linz, A-4040 Linz, Austria \\ R. Folk \\ Institute for Theoretical Physics, University of Linz, A-4040 Linz, Austria \\ Yu. Holovatch \\ Institute for Condensed Matter Physics, National Academy of Sciences of Ukraine, UA-79011 Lviv, Ukraine \\ Institute for Theoretical Physics, University of Linz,A-4040 Linz, Austria and \\ Ivan Franko National University of Lviv, UA-79005 Lviv, Ukraine \\ G. Moser \\ Institute for Physics and Biophysics, University of Salzburg, A-5020 Salzburg, Austria
}

\begin{abstract}
We consider the influence of quenched disorder on the relaxational critical dynamics of a system characterized by a non-conserved order parameter coupled to the diffusive dynamics of a conserved scalar density (model C). Disorder leads to model A critical dynamics in the asymptotics, however it is the effective critical behavior which is often observed in experiments and in computer simulations and this is described by the full set of dynamical equations of diluted model C. Indeed different scenarios of effective critical behavior are predicted.
\end{abstract}

Pacs numbers: 05.70.Jk; 64.60.Ht; 64.60.Ak

The critical behavior of pure systems might be changed by introducing imperfections like dilution, defects, etc. into a critical system. If such a change can be expected is answered by the Harris criterion [1] stating that a new diluted critical behavior appears if the specific heat of the pure system is diverging. The diluted critical behavior then has a nondiverging specific heat. Since the borderline value $n_{c}$ between a diverging and nondiverging specific heat at space dimensions $d=3$ lies between order parameter (OP) dimensions $n=1$ (Ising model) and $n=2$ (XY model) only the Ising case belongs to a new universality class. In consequence this result led to the conclusion that for the critical dynamics the coupling of conserved quantities to the $\mathrm{OP}$ is of no relevance [2, 3]. The argument was the following: For the critical dynamics of a relaxational model it was shown [4, 5, 6] that the coupling to a conserved density (e.g. the energy density) is relevant if the specific heat diverges. Due to dilution this is never the case and therefore the coupling is of no relevance. Therefore most of the papers considered only the relaxational dynamics of Ising systems [7, 8, 9, 10]

However this argumentation is based on the asymptotic properties of the diluted model. Experimental data and computer simulations made clear that in most cases one observes non-asymptotic critical behavior, described often by dilution dependent effective exponents (see e.g. 11, 12]). In such a case the Harris criterion does not hold and therefore one has to consider in the dynamics the coupling to the conserved density and its effects on the effective critical behavior. In addition one is not restricted to the Ising case since already in statics the effective critical behavior for $n>1$ is different from the pure case 12 .

There are two relevant parameters of model C: (i) the static coupling $\gamma$ of the OP to the conserved density and (ii) a dynamic parameter, the time scale ratio $w=\Gamma / \lambda$ where $\Gamma$ is the relaxation rate of the OP and $\lambda$ is the diffusion rate of the conserved density. From the renormalization group (RG) treatment of model $\mathrm{C}$ one knows that the one loop order does not give reliable results due to the stability of a fixed point with the time scale ratio $w=\infty$. In two loop (and higher) order it turns out that this fixed point is unstable and model $\mathrm{C}$ is characterized by strong and weak scaling regions for the dynamics at $d=3[5,6]$. Moreover it was shown that non-asymptotic effects are already present in model $\mathrm{C}$ [6]. In the following we will consider how these aspects are influenced by disorder.

Model C 4, 5] describes the relaxational dynamics of a system characterized by an $n$-component nonconserved OP $\vec{\varphi}_{0}(x, t)$ coupled to the diffusive dynamics of a conserved scalar density $m_{0}(x, t)$. The structure of the equations of motions is not changed by the presence of disorder. They read:

$$
\frac{\partial \vec{\varphi}_{0}}{\partial t}=-\stackrel{\circ}{\Gamma} \frac{\partial \mathcal{H}}{\partial \vec{\varphi}_{0}}+\vec{\theta}_{\varphi}, \quad \frac{\partial m_{0}}{\partial t}=\stackrel{\circ}{\lambda} \nabla^{2} \frac{\partial \mathcal{H}}{\partial m_{0}}+\theta_{m}
$$

where 0 or ${ }^{\circ}$ denote unrenormalized quantities. The stochastic forces in (1) satisfy the Einstein relations:

$$
\begin{aligned}
& <\theta_{\varphi_{i}}(x, t) \theta_{\varphi_{j}}\left(x^{\prime}, t^{\prime}\right)>=2 \stackrel{\circ}{\Gamma} \delta\left(x-x^{\prime}\right) \delta\left(t-t^{\prime}\right) \delta_{i j}, \quad(2) \\
& <\theta_{m}(x, t) \theta_{m}\left(x^{\prime}, t^{\prime}\right)>=-2 \grave{\lambda} \nabla^{2} \delta\left(x-x^{\prime}\right) \delta\left(t-t^{\prime}\right)(.3)
\end{aligned}
$$

Equilibrium is described by the static functional $\mathcal{H}$ of 


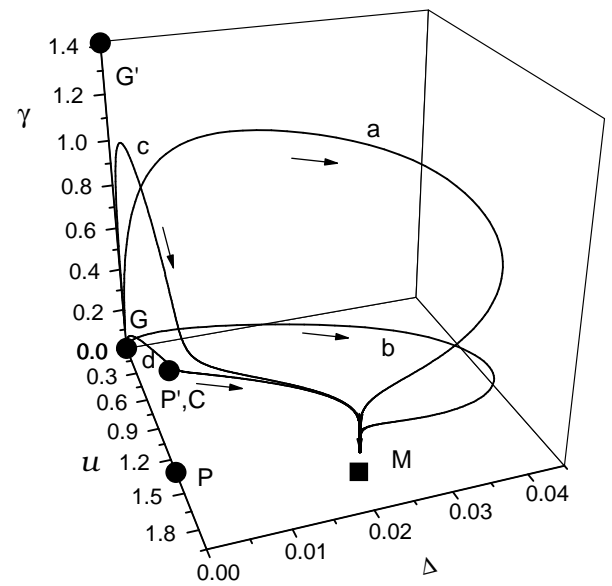

FIG. 1: Two loop flow of static parameters for $n=1$. The stable fixed point (full square) has a finite width $\Delta$ of the impurity distribution and leads to asymptotic exponents different from the pure case (unstable fixed points are indicated by full circles). Depending on the initial values of the renormalized couplings the effective exponents are quite different.

the disordered magnetic system

$$
\begin{aligned}
\mathcal{H} & =\int d^{d} x\left\{\left.\frac{1}{2} \stackrel{\tilde{r}}{\vec{\varphi}_{0}}\right|^{2}+V(x)\left|\vec{\varphi}_{0}\right|^{2}+\frac{1}{2} \sum_{i=1}^{n}\left(\nabla \varphi_{i, 0}\right)^{2}(4)\right. \\
& \left.+\frac{\tilde{u}}{4 !}\left|\vec{\varphi}_{0}\right|^{4}+\frac{1}{2} a_{m} m_{0}^{2}+\frac{1}{2} \delta m_{0}\left|\vec{\varphi}_{0}\right|^{2}-\stackrel{\circ}{h}_{m} m_{0}\right\},
\end{aligned}
$$

where $V(x)$ is an impurity potential which introduces disorder to the system, $d$ is the spatial dimension. It contains a coupling $\dot{\gamma}$ to the secondary density which can be integrated out. Thus static critical properties described by the functional (4) are equivalent to those of the functional $\mathcal{H}=\int d^{d} x\left\{\frac{1}{2} r\left|\vec{\varphi}_{0}\right|^{2}+V(x)\left|\vec{\varphi}_{0}\right|^{2}+\right.$ $\left.\frac{1}{2} \sum_{i=1}^{n}\left(\nabla \varphi_{i, 0}\right)^{2}+\frac{\grave{u}}{4 !}\left|\vec{\varphi}_{0}\right|^{4}\right\}$. The parameters $\stackrel{\circ}{r}$ and $\dot{u}$ are

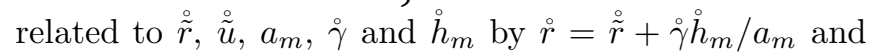
$\stackrel{\circ}{u}=\stackrel{\tilde{u}}{-}-3 \dot{\gamma}^{2} / a_{m} . \stackrel{\circ}{r}$ is proportional to the temperature distance from the mean field critical temperature, $\stackrel{i}{u}$ is positive. The properties of the random potential $V(x)$ are governed by a Gaussian distribution with width $\triangle$ $\left(<<V(x) V\left(x^{\prime}\right)>>=4 \AA \delta\left(x-x^{\prime}\right)\right.$, the double angular brackets means averaging over disorder). If $\dot{\gamma} \equiv 0$ equations (11) describes dynamical properties of a purely relaxational model (model A) in the presence of disorder [2].

We treat the critical dynamics of the disordered models within the field theoretical RG method [13], where the appropriate Lagrangians of the models are studied. The average over the random potential generates new terms in the Lagrangians with coupling $\stackrel{\Delta}{\Delta}$, which correspond to the static coupling terms in $\mathcal{H}$ generated by the disorder. The renormalization of the Lagrangian leads to the RG functions, describing the critical dynamics of our models. We use the minimal subtraction scheme with dimensional regularization to calculate these functions.

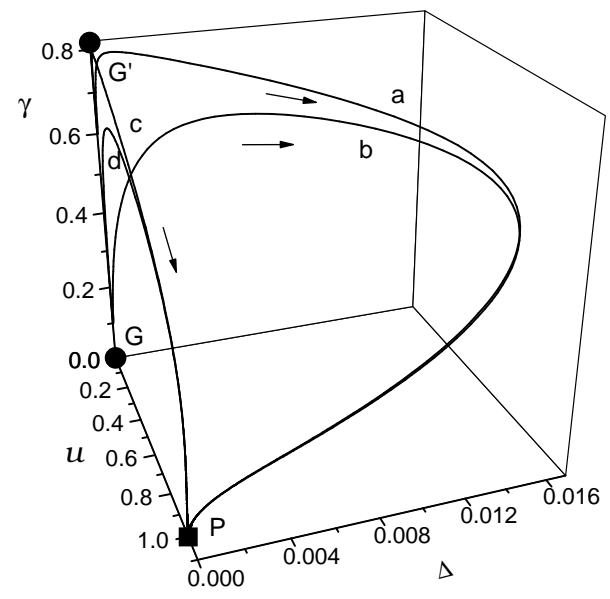

FIG. 2: Two loop flow of static parameters for $n=3$. The stable fixed point (full square) has a zero width $\Delta$ of the impurity distribution and leads to asymptotic exponents equal to the pure case (unstable fixed points are indicated by full circles). However depending on the initial values of the renormalized couplings the effective exponents are quite different and also depend on the inital value of the width $\Delta$.

For renormalization of the OP $\vec{\varphi}_{0}$, fourth-order cou-

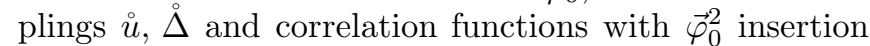
we introduce renormalization $Z$-factors as $\vec{\varphi}_{0}=Z_{\varphi}^{1 / 2} \vec{\varphi}$, $\stackrel{\circ}{u}=\mu^{\epsilon} Z_{\varphi}^{-2} Z_{u} u A_{d}^{-1}, \stackrel{\circ}{\Delta}=\mu^{\epsilon} Z_{\varphi}^{-2} Z_{\Delta} \Delta A_{d}^{-1}$, and $\left|\vec{\varphi}_{0}\right|^{2}=$ $Z_{\varphi^{2}}|\vec{\varphi}|^{2}$ ( $\mu$ is the scale, $\epsilon=4-d$ and $A_{d}$ is a geometric factor). Within dynamics renormalization factor for the OP kinetic coefficient $\stackrel{\circ}{\Gamma}=Z_{\Gamma} \Gamma$ is introduced. The $Z$ factors introduced so far are enough to renormalize the diluted model A. For model $\mathrm{C}$ one needs to introduce additional renormalization factors. The secondary density $m_{0}$ and coupling parameter $\dot{\gamma}$ are renormalized by $a_{m}^{1 / 2} m_{0}=Z_{m} m$ and $a_{m}^{-1 / 2} \dot{\gamma}=\mu^{\epsilon / 2} Z_{\varphi^{2}} Z_{m} \gamma A_{d}^{-1 / 2}$ with $Z_{m}^{-2}(u, \Delta, \gamma)=1+\gamma^{2} A_{\varphi^{2}}(u, \Delta)$. The kinetic coefficient $\lambda$ renormalizes as $a_{m} \stackrel{\circ}{\lambda}=Z_{m}^{2} \lambda$.

Defining the $\zeta$-functions as $d \ln Z^{-1} / d \ln \mu$, where $Z$ represents any renormalization factor, one obtains the flow equations for the renormalized static and dynamic parameters. The flow equations for $u$ and $\Delta$ decouple from the remaining parameters and are equal to expressions obtained for any $n$ in the diluted Ginsburg-LandauWilson (GLW) model [14]. For the additional static parameter $\gamma$ appearing in diluted model $\mathrm{C}$ we have

$$
l \frac{d \gamma}{d l}=\gamma\left[-\frac{\epsilon}{2}+\zeta_{\varphi^{2}}(u, \Delta)+\frac{1}{2} \gamma^{2} B_{\varphi^{2}}(u, \Delta)\right] .
$$

The flow parameter $l$ is related to the reduced temperature and is consequently a measure for the distance to the critical temperature. The function $\zeta_{c^{2}}(u, \Delta)$ is known from statics in the diluted model [14]. The function $B_{\varphi^{2}}(u, \Delta)$, which is defined by the additive renormalization of the specific heat within the GLW-model, in the diluted case reads $B_{\varphi^{2}}(u, \Delta)=n / 2+\mathcal{O}\left(u^{2}, \Delta^{2}, u \Delta\right)$.

The flow equation for the time scale ratio (we introduce 
TABLE I: Static and dynamic fixed points for $n=1$. Fixed points with $\rho^{*}=1\left(w^{*}=\infty\right)$ are always unstable and not shown. $\mathbf{M}$ is the stable fixed point of the diluted model $\mathrm{C}$.

\begin{tabular}{ccccc}
\hline \hline $\mathrm{FP}$ & $u^{*}$ & $\Delta^{*}$ & $\gamma^{*}$ & $\rho^{*}$ \\
\hline $\mathbf{G}$ & 0 & 0 & 0 & 0 \\
$\mathbf{G}^{\prime}$ & 0 & 0 & 1.414 & 0 \\
$\mathbf{P}$ & 1.315 & 0 & 0 & 0 \\
$\mathbf{P}^{\prime}$ & 1.315 & 0 & 0.458 & 0 \\
$\mathbf{C}$ & 1.315 & 0 & 0.458 & 0.266 \\
$\mathbf{M}$ & 1.633 & 0.021 & 0 & 0 \\
\hline \hline
\end{tabular}

$\rho=w /(1+w)$ instead $)$ is

$$
l \frac{d \rho}{d l}=\rho(1-\rho)\left[\zeta_{\Gamma}(u, \Delta, \gamma, \rho)-\gamma^{2} B_{\varphi^{2}}(u, \Delta)\right],
$$

where the dynamic $\zeta$-function $\zeta_{\Gamma}$ in two loop order reads

$$
\begin{aligned}
& \zeta_{\Gamma}(u, \Delta, \gamma, \rho)=\zeta_{\Gamma}^{(C)}(u, \gamma, \rho)+4 \Delta-\frac{n+2}{3} u \Delta+20 \Delta^{2} \\
& +2 \Delta \rho \gamma^{2}\left[3(1-\ln (1-\rho))+\rho \ln \frac{\rho}{1-\rho}-\frac{\rho}{1-\rho} \ln \rho\right] .
\end{aligned}
$$

The corresponding explicit two loop expression for the $\zeta$ function of model C, $\zeta_{\Gamma}^{(C)}(u, \gamma, \rho)=\zeta_{w}^{(C)}(u, \gamma, \rho)+n \gamma^{2} / 2$, is given in [6] (see $\zeta_{w}$ in Eq. (50) there). In these terms also the pure model $\mathrm{A}$ terms are included, which taken together with the last three terms in the first line of Eq. (77) recover the $\zeta$-function for the diluted model A 2, 8]. The zeros of the right hand sides of Eqs.(5), (6), and the corresponding equations for $u$ and $\Delta$ give the possible fixed points.

It turns out that for all values of $n$ the stable fixed point value $\gamma^{\star}=0$, and the well-known asymptotic static results are reproduced. Thus the flow in the space of the static couplings $u, \Delta$ and $\gamma$ for $n<n_{c}<2$ looks like the flow for $n=1$ (see Fig. 1) whereas for $n>n_{c}$ it looks like the flow for $n=3$ (see Fig. 22). The fixed points for $n=1$ are indicated in Tab. प Only the mixed fixed point ( $\mathbf{M}$ in Fig (1) is stable. However depending on the initial conditions a rich crossover behavior is observed.

TABLE II: Stability exponents according to the fixed points in Tab凹 for $n=1$.

\begin{tabular}{ccccc}
\hline \hline $\mathrm{FP}$ & $\omega_{u}$ & $\omega_{\Delta}$ & $\omega_{\gamma}$ & $\omega_{\rho}$ \\
\hline $\mathbf{G}$ & -1 & -1 & -0.5 & 0 \\
$\mathbf{G}^{\prime}$ & -1 & -1 & 1 & 0 \\
$\mathbf{P}$ & 0.566 & -0.105 & -0.053 & 0.052 \\
$\mathbf{P}^{\prime}$ & 0.566 & -0.105 & 0.105 & -0.053 \\
$\mathbf{C}$ & 0.566 & -0.105 & 0.105 & 0.041 \\
$\mathbf{M}$ & 0.494 & 0.194 & 0.0018 & 1.139 \\
\hline \hline
\end{tabular}

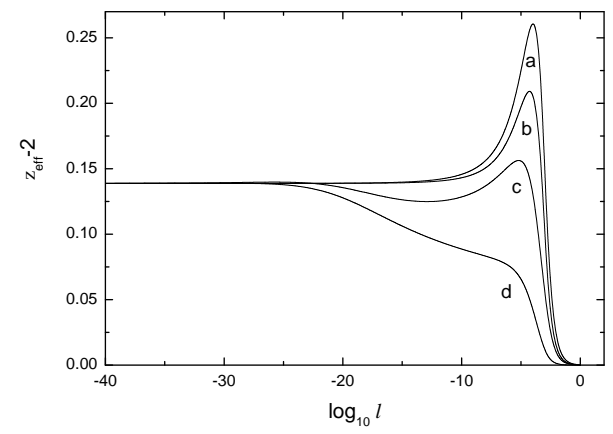

FIG. 3: Effective dynamical exponent for $n=1$ for different initial conditions. The lines correspond to the static flows shown in Fig 1

The same is true for $n=3$ although now the pure fixed point ( $\mathbf{P}$ in Fig. $\mathbf{2})$ is stable.

The stable fixed points are found by calculating the stability matrix and its eigenvalues $\omega_{i}$ ( $i$ represents $u, \Delta$, $\gamma$ or $\rho$ ) . They govern the "velocity" of the flow near the fixed points. A small stability exponent at the stable fixed point indicates a slow approach of the asymptotic behavior. This is the case for all $n$ and can be seen from Tab【IIfor $n=1$. It is the slow approach in the $\gamma$ direction which characterizes the static flow within model C (see the extremely small values $\omega_{\gamma}=0.0018$ for $n=1$ [at the fixed point $\mathbf{M}$ in Tab $\Pi$ and for $n=3$ still $\omega_{\gamma}=0.1109$ ). In addition to the small values of the static stability exponents near the fixed points one has also small values $\omega_{\rho}$ coming from the dynamic parameter $\rho$ (however only near unstable fixed points). Thus one expects depending on the initial values of static and dynamic parameters a complex behavior in the non-asymptotic region. The effective dynamical exponent is of special interest here, it is found by inserting the solutions of the flow equations into the expression

$$
z_{e f f}(l)=2+\zeta_{\Gamma}(u(l), \Delta(l), \gamma(l), \rho(l)) .
$$

One reaches the universal asymptotic value $z$ when the flow comes very near the stable fixed point. One observes

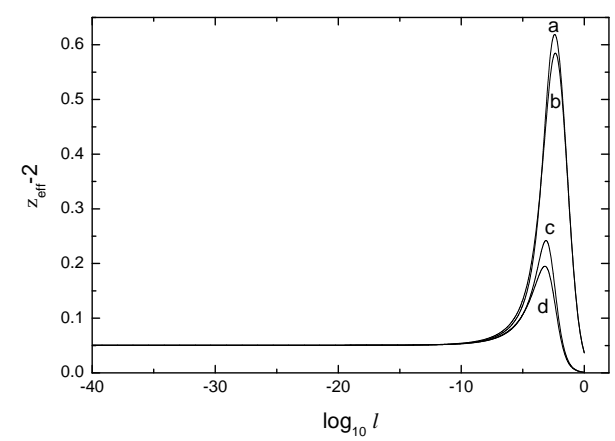

FIG. 4: Effective dynamical exponent for $n=3$ for different initial conditions. The lines correspond to the static flows shown in Fig[1] 


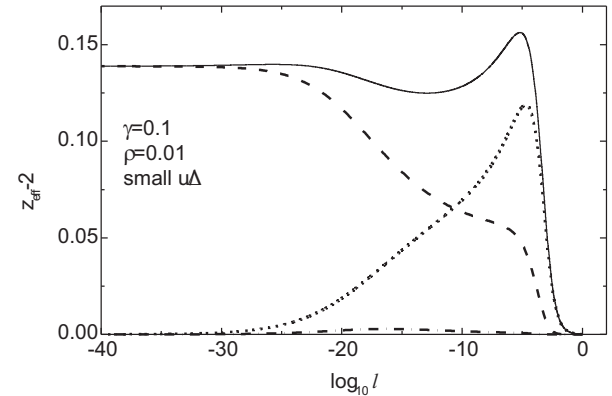

FIG. 5: Different contributions to the effective dynamical critical exponent for $n=1$. The solid line represents the total $z_{\text {eff }}$, for the other lines see the text.

that the stable fixed point value of the time ratio is always zero, independently of the specific heat exponent value of the pure model. Consequently, the results for model A are recovered in the asymptotics (either in the diluted universality class for $n<n_{c}$ or in the pure model A universality class for $n>n_{c}$ ). This combines with the fact that even in the region where dilution changes the static critical behavior the stable fixed point value of $\gamma^{*}$ is always zero. In consequence the secondary density is for all $n$ asymptotically decoupled and has an asymptotic dynamical exponent $z_{m}=2$.

The non-asymptotic behavior is however quite different as can be seen from Figs. 3 and 4 Due to the static non-asymptotic behavior also the dynamics is dominated by different non-asymptotic effects. This can be seen by comparing different $z_{e f f}(l)$ for different initial conditions. The curves $\mathrm{a}, \mathrm{b}$, and $\mathrm{c}$ in Fig. 1 and all curves in Fig. 2 reach large values of the coupling $\gamma$ and/or $\Delta$ and this leads to the typical maximum in the effective exponents independent of the initial value of $\rho$ (for the statics see e.g. [12]). However an additional fixed point
$\mathbf{P}^{\prime}$ is present at $n=1$. This leads for curve d in Fig. 1 almost to a plateau of $z_{\text {eff }}$ at its value for the unstable fixed point $\mathbf{P}^{\prime}$. This plateau is more pronounced when the flow comes nearer to $\mathbf{P}^{\prime}$ where it stays longer because of the small transient exponent $\omega_{\rho}$. For curve c both effects (the maximum and the effect of fixed point $\mathbf{P}^{\prime}$ ) are combined leading to the minimum in $z_{\text {eff }}$. Consider now the contributions to the effective dynamical critical exponent $z_{\text {eff }}$ of different origin: (i) from the terms already present in model A (dashed curve in Fig. 5), (ii) from terms present in pure model $\mathrm{C}$ only (short dashed curve) and (iii) and from terms present in the diluted model $\mathrm{C}$ only (dashed-short dashed curve). The above contributions may add up to almost the asymptotic value of the exponent although the parameters are far away form their asymptotic values. This is an important point since the appearance of an asymptotic value in one physical quantity does not mean that other quantities have also reached the asymptotics. This is due to the different dependence of physical quantities on the model parameters. Another special feature of the diluted model $\mathrm{C}$ is that already in one loop order one observes qualitatively the same behavior as in two loop order of course with changed values for the exponents and the borderline value $n_{c}$, which in one loop is at $n_{c}=4$.

In concluding we remark that contrary to the general belief the coupling of a conserved density to the order parameter is relevant for the calculation of the dynamical critical behavior of diluted systems since this coupling is important to describe non-asymptotic effects. These effects have been seen in the experiments on physical systems [12, 15] as well as in Monte Carlo simulations [16].

We acknowledge support from the Fonds zur Förderung der wissenschaftlichen Forschung (project P16574)
[1] A. B. Harris, J. Phys. C: Solid State Phys. 7, 1671 (1974).

[2] I. D. Lawrie, V. V. Prudnikov, J. Phys. C: Solid State Phys. 17, 1655 (1984).

[3] U. Krey, Phys. Lett. 57A, 215 (1976).

[4] B. I. Halperin, P.C.Hohenberg, and Shang-keng Ma, Phys. Rev. B 10, 139 (1974).

[5] R. Folk and G. Moser, Phys. Rev. Lett. 91, 030601 (2003).

[6] R. Folk, G. Moser, Phys. Rev. E 69, 036101 (2004).

[7] G.Grinstein, S.-k. Ma, and G. Mazenko, Phys. Rev. B 15, 258 (1977).

[8] V. V. Prudnikov, A. N. Vakilov, Sov. Phys. JETP 74, 990 (1992).

[9] K. Oerding and H. K. Janssen, J. Phys. A: Math. Gen. 28, 4271 (1995).

[10] H. K. Janssen, K. Oerding, and E. Sengenspeick, J. Phys. A: Math. Gen. 28, 6073 (1995).

[11] For the recent reviews see e.g.: R. Folk, Yu. Holovatch, T. Yavors'kii, Phys. Rev. B 61, 15114 (2000); R. Folk,
Yu. Holovatch, Physics-Uspiekhi, 46, 169 (2003); A. Pelissetto, E. Vicari, Phys. Rep. 368, 549 (2002).

[12] A. Perumal et al. Phys. Rev. Lett. 91, 137202 (2003); M. Dudka, Yu. Holovatch, R. Folk and D. Ivaneiko, J. Magn. Magn. Mater. 256, 243 (2003).

[13] R. Bausch, H. K. Janssen, and H. Wagner, Z. Phys. B 24, 113 (1976).

[14] J. Kyriakidis, D. J. W. Geldart, Phys. Rev. B 53, 11572 (1996).

[15] P. H. Barrett, Phys. Rev. B 34, 3513 (1986); D. P. Belanger, B. Barago, V. Jaccarino, A. R. King, C. Lartigue, and F. Mezei, J. Phys. (Paris) Colloq. 49, C8-1229 (1988); N. Rosov, C. Hohenemser, M. Eibschütz, Phys. Rev. B 46, 3452 (1992).

[16] H. G. Ballesteros et al. Phys. Rev. B 58, 2740 (1998); G. Parisi, F. Ricci-Tersenhi, and J. J. Ruiz-Lorenzo, Phys. Rev. B 60, 5198 (1999). 